\title{
Complex systems approach for sports injuries: moving from risk factor identification to injury pattern recognition-narrative review and new concept
}

\author{
N F N Bittencourt, ${ }_{1}^{1}$ W H Meeuwisse, ${ }^{2}$ L D Mendonça, ${ }^{3}$ A Nettel-Aguirre, ${ }^{4}$ \\ J M Ocarino, ${ }^{5}$ S T Fonseca ${ }^{5}$
}

'Physical Therapy Department, Minas Tenis Clube and Uni-BH University, Minas Gerais, Belo Horizonte, Brazil

${ }^{2}$ Sport Injury Prevention Research Centre, Faculty of Kinesiology, University of Calgary, Alberta, Canada ${ }^{3}$ Physical Therapy Department, Universidade dos Vales do Jequitinhonha e Mucuri, Minas Gerais, Diamantina, Brazil ${ }^{4}$ Departments of Paediatrics and Community Health Sciences, University of Calgary, Alberta, Canada

${ }^{5}$ Graduate Program in

Rehabilitation Science, Physical Therapy Department,

Universidade Federal de Minas Gerais, Minas Gerais,

Diamantina, Brazil

\section{Correspondence to} Dr ST Fonseca, Graduate Program in Rehabilitation Science, Physical Therapy Department, Universidade Federal de Minas Gerais, Minas Gerais, Avenida Presidênte Antônio Carlos, 6627-Pampulha, Belo Horizonte-MG 31270-901, Brazil; sfonseca@ufmg.br

Accepted 18 June 2016 Published Online First 22 July 2016

\section{ABSTRACT}

Injury prediction is one of the most challenging issues in sports and a key component for injury prevention. Sports injuries aetiology investigations have assumed a reductionist view in which a phenomenon has been simplified into units and analysed as the sum of its basic parts and causality has been seen in a linear and unidirectional way. This reductionist approach relies on correlation and regression analyses and, despite the vast effort to predict sports injuries, it has been limited in its ability to successfully identify predictive factors. The majority of human health conditions are complex. In this sense, the multifactorial complex nature of sports injuries arises not from the linear interaction between isolated and predictive factors, but from the complex interaction among a web of determinants. Thus, the aim of this conceptual paper was to propose a complex system model for sports injuries and to demonstrate how the implementation of complex system thinking may allow us to better address the complex nature of the sports injuries aetiology. According to this model, we should identify features that are hallmarks of complex systems, such as the pattern of relationships (interactions) among determinants, the regularities (profiles) that simultaneously characterise and constrain the phenomenon and the emerging pattern that arises from the complex web of determinants. In sports practice, this emerging pattern may be related to injury occurrence or adaptation. This novel view of preventive intervention relies on the identification of regularities or risk profile, moving from risk factors to risk pattern recognition.

\section{INTRODUCTION}

Injury prediction is one of the most challenging issues in sports and a key component for injury prevention, since the successful identification of injury predictors forms the basis for effective preventive measures. ${ }^{1}$ Traditionally, scientific investigations have assumed a reductionist view in an attempt to understand sports injuries. According to this approach, the phenomenon (injury) has been reduced into units and explained by rational deduction. ${ }^{3}$ The sports injury literature has revealed important injury predictors by means of typical statistics tools, such as logistic regression. However, for some injuries (eg, hamstring strain and patellar tendinopathy (PT)), these techniques have not yet yielded consistent identification of risk factors. ${ }^{4} 5$ Unfortunately, these inconsistencies show that the majority of the human health conditions are complex. In this sense, we need a broader approach to better understand the complex relationships between risk factors/predictors and injuries. ${ }^{2-4} 6-8$

To address this issue, Meeuwisse et al ${ }^{9}$ developed a dynamic, recursive model for risk and causes of sports injuries, considering that the injury has a non-linear behavior. This model brought many advances to the understanding of sports injuries aetiology, because it assumes that there may be recurrent changes in susceptibility to injury along the participation in sports, and the primary risk factors exposure can produce adaptations and continuously change the risk. Despite the recognition of the non-linear and recursive characteristics of sports injuries, the model described by Meeuwisse et $a l^{9}$ was not sufficient to address the complex interactions among several factors.

The multifactorial and complex nature of sports injuries arises not from the linear combination of isolated and predictive factors, but from the interaction among what Philippe and $\mathrm{Mansi}^{8}$ called 'the web of determinants'. These determinants may be linked to each other in a non-linear manner, in the sense that small changes in a few determinants can lead to large and, sometimes, unexpected consequences. ${ }^{6}{ }^{10}$ We propose that, to fully uncover this complex nature of sports injury aetiology, a complex systems approach is necessary. This approach rests on identifying interactions and clarifying how these interactions contribute to the emergence of sports injuries. ${ }^{10}{ }^{11}$ In addition, it allows seeking regularities (see explanation in the characteristics of a complex system section), by means of pattern recognition techniques, ${ }^{12}$ that enable the identification of the risk profile for an athlete or group. Thus, the aim is to introduce a complex system model for sports injuries and to demonstrate how the implementation of complex system thinking may allow us to better address the dynamic nature of the sports injuries aetiology.

This conceptual paper was developed on the basis of reflections on the difficulties related to sports injuries prediction and how different areas are trying to solve similar problems. These reflections and subsequent exhaustive literature review led to several discussions among the authors that resulted in this work. To facilitate the understanding of our proposition, this paper is organised into six sections: introduction; a paradigm shift: from reductionism to complexity describing the reductionist and complexity paradigms; characteristics of 
a complex system; a complex model for sports injury which assumes sports injury is an emergent phenomenon that arises from interactions within a web of determinants; methodological implications of using a complex systems approach and applying a complex model for sports injury.

\section{A PARADIGM SHIFT: FROM REDUCTIONISM TO COMPLEXITY}

The simplification of complex problems into basic units is the classical science method of analysis in the reductionism paradigm. $^{2} 48$ This approach has focused on identifying isolated factors that are frequently assumed as causes of injuries or diseases. ${ }^{8} 12$ In many aspects, this has been a successful approach, such as establishing a strong causal connection between smoking and lung cancer. ${ }^{13}$ Reductionism has thus helped identify some apparently existing linear relationships. ${ }^{8}$ Linearity assumes that the outcome is somehow related to the sum of the system's units and that it can be predicted (although subject to random errors) by looking for these direct relationships. ${ }^{78}$ However, this linear association characterises a singular circumstance of relationship: the closer the exposure is to the end point, the greater is its impact. ${ }^{8}$ A typical example in sports injury is the identification of dynamic knee valgus (DKV) as a risk factor for ACL rupture. ${ }^{14}$ It has been identified that female athletes who demonstrated knee valgus during landing, $50 \mathrm{~ms}$ after initial contact, are five times more prone to injuring their ACL than male athletes (relative risk $(R R)=5.3, p=0.002) .{ }^{15}$ Since the scales we use measure the triggering factors that are proximal to end point outcome, such linear relationships can be revealed. ${ }^{8}$ The concern here is that this relationship represents only a segment of the total picture. ${ }^{8}$

The picture is different when the initial event (exposure) is distant or when there are several factors at multiple levels (biomechanical, behavioural, physiological, etc) that can influence each other. For instance, restriction in ankle dorsiflexion range of motion (ROM) and training load are found to be risk factors (although inconsistent) for PT. ${ }^{5}$ However, in some cases, restricted ankle dorsiflexion ROM only will be relevant in the presence of high training load. This last risk factor will influence the former because high frequency of vertical jump increases the total mechanical energy reaching the tissues, which should be dissipated by the hip, knee and ankle. In the presence of restricted ankle dorsiflexion ROM, this energy may not be properly dissipated at the ankle and could overload the patellar tendon. ${ }^{16}$ Additionally, the amount of training load could be modulated by the excessive demands of external sponsors or coaches' training philosophy. This chain of possible related events establishes a complex web of injury determinants, which requires a complexity approach to be better understood. ${ }^{8}$ Historically, complexity thinking was influenced by developments in several areas, such as information theory, cybernetics and systems theory, and, in turn, has been adopted by many disciplines. $^{6} 10 \quad 17-20$ Biology and medicine recognise that living organisms are understood as complex systems characterised by multiple interactions among units, self-organisation, nonlinearity and emergent properties. ${ }^{6}{ }^{21}$ More recently, a complex systems approach has been used in epidemiology to improve the knowledge about multilevel causes of health and diseases. ${ }^{12}$

Prediction of complex problems, such as financial crises, ${ }^{20}$ diseases, $^{12} 21$ athlete high performance ${ }^{22}$ and, potentially, sports injury should involve the identification of the complex web of determinants. In sports, the focus should be on identifying the stable (sometimes complex) relationships among injury determinants that support the emergence of injuries and not on the contribution of isolated factors. ${ }^{11}$ We need to be aware that when we reduce a complex problem into its units, the relationships are neglected and prediction may not be possible. ${ }^{11}{ }^{23}$ Sports injury will be better understood if we recognise the frequent patterns of interaction among multilevel risk factors instead of focusing on identifying risk factor at the units level. ${ }^{11}$ Accordingly, Mendiguchia et $a l,{ }^{4}$ Quatman et $a l^{2}$ and Hulme and Finch ${ }^{11}$ have proposed a paradigm shift in sports injury research. These authors emphasised that the literature is limited to consistently identifying predictive factors, because current research methods based on unidirectional and analytical approaches neglect the multifactorial and complex conditions for sports injuries emergence. ${ }^{11}$ The lack of proper methodological approaches limits our ability to identify effective preventive strategies, as we frequently fail to properly recognize the web of injuries determinants. Consequently, some injuries rates, such as hamstring strain, have not improved over the past three decades. ${ }^{24}$

The complexity paradigm contributes to examining sports injury in terms of relationships rather than factors. However, complexity paradigm should not completely replace reductionism. ${ }^{11} 12$ It is possible to argue that complexity paradigm is an advance from reductionism. Although linear approaches may facilitate the identification of triggering factors that are close to the sports injury mechanism, they fail to recognise the inherent non-linearity of biological processes that are related to sports injuries. This interrelationship explains why the search for cause-effect relationships will always be problematic. Given the complexity of the sports injuries process, the future of aetiology research must advance towards the integrated view of the complex systems approach.

\section{CHARACTERISTICS OF A COMPLEX SYSTEM}

The understanding about interactions is the cornerstone of the complex systems approach. von Bertalanfly ${ }^{25}$ has defined a complex system as a whole with units (parts) that interact with each other. However, according to Rosen, ${ }^{26}$ these interactions are complex in the sense that the participating units are modified by the occurring interactions or due to the fact that new and unpredictable units emerge during the process. When units A and $\mathrm{B}$ interact, the behaviour of $\mathrm{A}$ in interaction with $\mathrm{B}$ is different from the behaviour of A alone. ${ }^{25}{ }^{26}$ Regarding sports injuries, the athlete should be analysed as a complex system and the research focus would be on how relationships between units (ie, biomechanical, behavioural, physiological and psychological) give rise to the collective behaviour of the athlete and how the athlete interacts and forms stable relationships (regularities) with his/her environment.

Complex systems are dynamic, open systems with inherent non-linearity due to the existence of recursive loops and complex interactions among units, which spontaneously organise themselves to generate emerging properties that cannot be deduced solely from their original properties (self-organisation). ${ }^{3}{ }^{10}$ Such systems are posed with some level of uncertainty as the multiple levels and scales (size and time) of the interacting units are frequently unknown or not directly observable. ${ }^{17}$ These characteristics are explained in more detail below.

\section{Open system}

In physics, all living organisms are open systems, as they exchange matter and energy with the environment, without losing their identity. ${ }^{7} 27$ In contrast, a system is closed if the influence of the environment on it can be ignored, as no matter or energy enters or leaves the system. ${ }^{27}$ To better distinguish closed and open systems, the concept of equifinality must be introduced. $^{27}$ Equifinality means that there are many diverse ways in which the same emergent outcome can be generated. ${ }^{27}$ 
This type of behaviour is frequently seen in sports injuries, in which different relations among risk factors produce the same injury (emergent outcome; see example in applying complex model for sports injury section). Open systems fully interact with the environment and evolve over time, producing multiple pathways to similar outcomes. ${ }^{7}$

\section{Inherent non-linearity}

Complex systems exhibit non-linear behaviours that are uncertainly related to the input provided. ${ }^{37} \mathrm{~A}$ linear system is characterised by the superposition principle. This principle implies that the observed final system's global behaviour is directly related to the sum of the contribution of its individual parts. ${ }^{7} 8$ However, the superposition principle cannot be applied to nonlinear systems, because inputs are not proportional to the output. ${ }^{719}$ Typically, large changes in one variable do not necessarily produce a large effect on the outcome. Conversely, small changes may produce a large and sometimes unexpected effect on the outcome. ${ }^{19}$ The process related to abrupt changes in the system's configuration that is observed in non-linear systems is termed 'emergence'. ${ }^{6}$ The emergent properties of a complex system are the result of interactions among units and are not equivalent to units themselves. ${ }^{28}$

Injury prediction in sports is less straightforward and frequently dependent on dose-response relationships. For example: DKV (high frontal plane knee angle) has been associated with hip abductor weakness. ${ }^{29}{ }^{30}$ In a linear view, one could assume that the weaker the hip abductors, the larger the DKV. However, evidence has shown a non-linear relationship between hip muscle strength and $\mathrm{DKV}^{30}$ When participants have high hip internal rotation stiffness (as in the case of hip retroversion), even a high strength level of the hip abductor is not capable of preventing DKV. ${ }^{30}$ There are several examples in the sports literature in which a variable is important but not determinant for injury occurrence. ${ }^{31-33}$ Therefore, the nonlinear nature of sports injury precludes us from finding the expected relationships among predictors and outcomes.

\section{Recursive loop}

Another essential characteristic of a complex system is the existence of a recursive loop (feedback), in which the output is reprocessed and becomes new input for the system. ${ }^{7} 9$ Interacting units in a system create overall patterns, and these overall patterns in turn cause the interacting units to change or adapt. $^{20}{ }^{26}$ For example, there is a recursive loop among the hypothalamus, pituitary and ovary. The hypothalamus releases a gonadotropin-releasing hormone, which stimulates the pituitary gland to produce the follicle-stimulating hormone (FSH) and luteinising hormone (LH). The FSH and $\mathrm{LH}$ in turn stimulate the ovary, resulting in the production of female hormones, which further controls the secretion by the hypothalamus and pituitary. The hypothalamus, pituitary and ovary interactions are so intricate that any dysfunctional activity at any level in the recursive loop can result in menstrual dysfunction. ${ }^{34}$ This 'global to local' recursive loop indicates that the global pattern emerges from interactions among local units, and this global pattern influences and constrains the local units' interactions (eg, the enslaving principle described by $\mathrm{Haken}^{28}$ ). Recognising the existence of recursive loops should remind us that, after an injury occurrence, the system might change in an unpredictable way. $^{7}{ }^{9}$ In this case, the previous states of the so-called injury predictors are changed as to no longer exhibit the same relationship to the outcome or even be present at all.

\section{Self-organisation (regularities and emerging patterns)}

The non-linear interactions among individual units of a system result in the emergence of properties that could not be predicted on the basis of the behaviour of the individual units alone. ${ }^{67} 19$ These emergent properties are the result of a process called self-organisation, which is governed by universal laws. ${ }^{28}$ These universal laws, or rules, enable self-organisation, creating spontaneous occurrence of order within the system. ${ }^{7}{ }^{28}$ Owing to self-organisation, the overall behaviour of the system is not proportional to the individual behaviour of the units, as it is through the units' interactions (cooperation) that the new pattern is produced. ${ }^{78}$ When certain critical values of the system's units are reached, specific configurations of the interacting units will produce observable regularities. In sports injuries, these regularities are regularly occurring patterns that are related to the final emerging outcome. Observed regularities could be classified as an injury risk or protective profile.

In complex systems, since the existing units (risk factors) and their interactions are frequently unknown and their direct relationship with the outcome is non-existent (or weak), ${ }^{33}$ the only means to infer system dynamics is by means of observing its regularities (risk profile). As with the weather, the occurrence of certain regularities may help scientists to predict the occurrence of certain events. For example, the irregularly occurring El Niño phenomenon is a kind of regularity (identified as a specific variation in sea surface temperature) that is related to the occurrence of floods and droughts in many regions. In sports, the complex nature of the mechanisms involved with the emergence of a given injury can be compressed into the simpler relationship between the observable regularity and the emerging outcome (eg, injury or adaptation).

\section{Uncertainty}

In the emergence of many phenomena, prediction cannot be made by means of the identification of the relationship between cause and effect. ${ }^{8}$ In those instances, prediction relies only on the identification of the probability of the occurrence of emerging phenomena. This uncertainty in the behaviour of complex systems is the result of self-organisation and existence of nonlinear relationships within the system that lead to many pathways to environmental adaptation. ${ }^{3}{ }^{35}$ Despite the lack of full predictability, some approaches can be used to reduce uncertainty, such as identifying recurring patterns that consider context and history. ${ }^{3} 735$ Relatively simple patterns have been shown in biological systems (such as beat-to-beat variation in heart rate), social systems (nurse's staffing pattern on a hospital ward) and financial markets (exchange rates) that are related to the outcome of interest. ${ }^{3} 1920$

The aforementioned characteristics, such as open system, nonlinearity, recursive loop, self-organisation and uncertainty, support the understanding of sports injury as a complex phenomenon. To facilitate this understanding, a complex model for sports injury is required.

\section{A COMPLEX MODEL FOR SPORTS INJURY}

We propose an alternative model for sports injury that might serve as a new perspective for understanding injury aetiology. This model is graphically represented in figure 1. Interacting units of a complex system result in a web of determinants, in which the units interact with each other in unpredictable and unplanned ways (frequently unknown). ${ }^{78}$ These complex interactions arise from the system's own history and form observable regularities (risk or protective profile), which in turn emerge as 


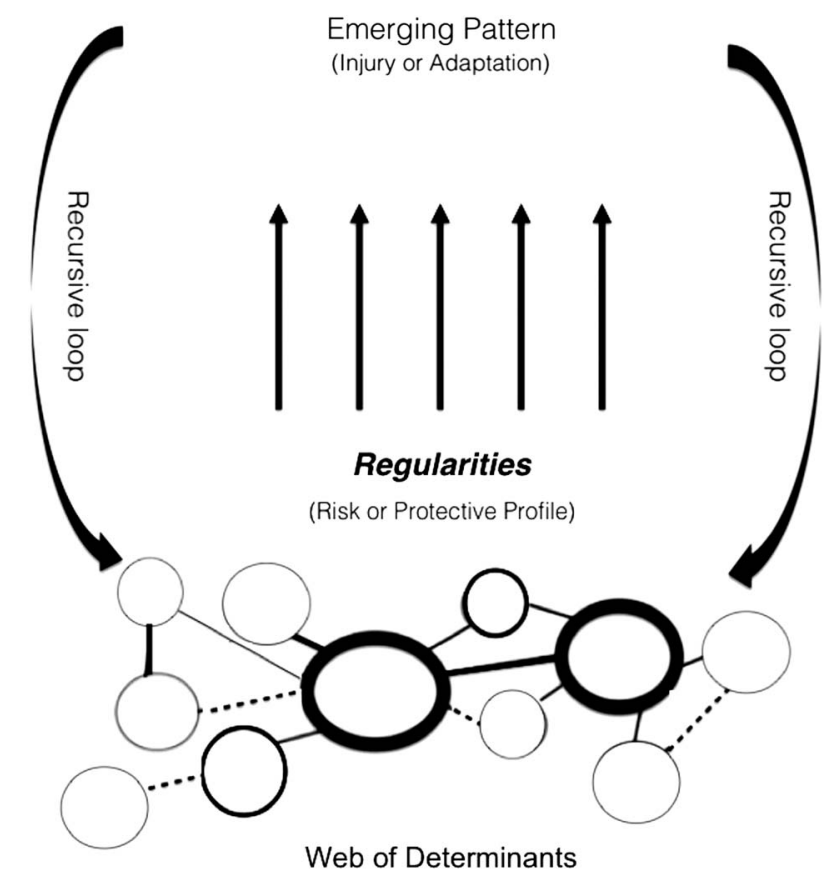

Figure 1 Complex model for sports injury. The group of variables at the bottom makes up the web of determinants, which is composed of contributing units with different weights. Variables circled by darker lines have more interactions than variables circled by lighter lines and exert a greater influence on the outcome. Dotted lines represent a weak interaction and thick lines represent a strong interaction between variables. Arrows indicate the relationship between the observable regularities, which captures the risk/protective profile, and the emerging outcome (figure adapted from ref. 47).

a global pattern (injury or adaptation). This emerging pattern constrains the interactions among the system's units (the model's recursive loops) and will dynamically shape the existing interactions. In this case, the web of determinants may change according to the outcome they produce.

Complexity requires accepting some level of uncertainty (despite a possible determinism) in regard to the occurrence of the phenomena. ${ }^{17}$ As in a weather forecast, prediction is improving over time, but errors are always a possibility. To face this uncertainty problem, this paper proposes that sports injury research needs to concentrate on analysing the observable regularities that arise from the existing simple and complex interactions among the elements of the web of determinants and not the units themselves (risk factors). Consequently, to identify the overall functioning of a system in relation to its principles and improve prediction, it is necessary to apply complex system methods.

\section{METHODOLOGICAL IMPLICATIONS OF USING A COMPLEX SYSTEMS APPROACH}

Improvements in sports injuries prediction, as well as in prevention, depend on coherence among the phenomena of interest (sports injuries as an emergent event), philosophical paradigm (complexity) and methods of analysis. In this sense, non-linear and complex system approaches should be explored. At the methodological level, complexity assumes that prediction relies on probability and not on straightforward causality (even in deterministic systems, the lack of knowledge about the initial conditions of the existing elements leads to a great degree of uncertainty). To improve our prediction capability, we need to capture stable interactions among units (risk factors) and the observable regularities (profiles). Economists have considered characteristics of individuals and of global societal dynamics to show how different stock market trading strategies can emerge from simple rules. ${ }^{20}$ Likewise, meteorologists have been using complex system computation technics to improve the weather forecast. ${ }^{36}$

Advances in predicting complex problems, such as financial crises and sports performance, ${ }^{20} 22^{37}$ have been possible by using classification methods in the statistical learning arena like neural networks (eg, Self-Organizing Feature Maps (SOFM)). ${ }^{38}$ Pfeiffer and Hohmann ${ }^{22}$ found that sports talent development outcome was better predicted by means of the non-linear method of neural network (SOFM) than by linear methods such as discriminant analysis (DA). Percentages of total correctly predicted cases by SOFM were $87.9 \%$ vs $69 \%$ by DA. The authors highlighted that neural networks are able to recognise global patterns of different talent development and that SOFM are worthwhile tools in the analysis of non-linear sports talent processes. ${ }^{22}$

In medicine, the array of recursive partitioning based methods such as classification and regression trees (CART), random forests and boosting, which allow non-linear interactions among predictors, as well as depict and make use of these interactions, have been successful in identifying the subset of risk and predictive factors to explain different outcomes. ${ }^{30} 3940$ Bittencourt et $a l^{30}$ have used CART to predict high frontal plane knee angle (DKV) during landing from a vertical jump. ${ }^{30}$ They have shown that the contribution of the shank-forefoot varus alignment to the occurrence of high frontal knee angle depended on the presence of hip abductor weakness. Varus alignment and abductor weakness were defined in terms of cut-off points, in the sense that having values above or below the cut-offs did not increase or decrease the probability of the occurrence of DKV (non-linearity). ${ }^{30}$ Recently, an agent-based model (ABMs) has been used in epidemiology to capture complex relationships not identified by logistic regression. ${ }^{41}$ Although these methods are not commonly introduced in statistical courses, computer programs are becoming easily available on the internet. For example, the software NetLogo (written in java) allows modelling ABMs, by means of simple commands, and enables the construction of webs of interrelated units. ${ }^{42} \mathrm{We}$ proposed that these statistical models should be adopted in sports science to investigate processes involving interactions among different predictors and identify dynamic interactions, with the final purpose of prediction.

Another statistical approach that might be an appropriate candidate to reveal possible interacting predictors of sports injury is machine/statistical learning. Statistical learning has been expressed as the overlapping area of statistics, computational data mining, artificial intelligence and engineering. ${ }^{38}$ Much of its use has been in the area of supervised learning, in which an observed outcome is investigated by looking at the patterns of other factors, in an attempt to learn which pattern leads to that specific outcome. Many of the statistical learning tools do not necessarily yield only one coefficient or rate of change for one specific predictor. For example, in the case of CART, a predictor may play a role in several splits of different branches, depending on the other predictors higher up in the tree. ${ }^{30}$

$\mathrm{Up}$ to the recent development of Bayesian Additive Regression Trees, ${ }^{43}$ the usual expected and idolised ' $p$ value' that accompanies the regression models, or any other form of inferential confidence or probability value on point estimates, is not part of the output obtained from recursive partitioning models. Owing to these two main issues, the paradigm has to be challenged to move into the arena of prediction rather than on examining the impact of a factor and on relying on other measures of 'success' such as misclassification rates. Statistical 
learning tools may be more accurate in predictions than the usual linear models, and the amount of assumptions could be lessened. ${ }^{38}$ Accordingly, we need to ask ourselves, without taking away the epidemiological importance of identifying risk factors and their potential modifiability, whether we are aiming at prediction or solely at finding relationships. In order to catch patterns and be able to prevent, we need to predict.

Apart from statistical issues, we also have to methodologically incorporate the complex system thinking. Meeuwisse et $a l^{9}$ highlighted the dynamic nature of risk factors, as the pattern of change in one variable (ie, change of strength during a season) could influence injury risk more than its absolute value in one point in time. Such a concept requires examining risk factor change longitudinally over a period of time, in order to incorporate the history of the event. For this reason, study designs and statistical analyses must consider the existence of complex interactions and changing risks. In practice, assessing risk profile should occur along the season, not only in the preseason, and the analyses should focus on the changing web of determinants that lead to an emergent injury.

\section{APPLYING COMPLEX MODEL FOR SPORTS INJURY}

Sports injury is a complex emergent phenomenon ${ }^{11}$ and needs to be seen through a 'lens of complexity'. In this case, we should seek to identify features that are present in complex systems: (1) the pattern of relationships (interactions) between units (determinants); (2) the regularities (profiles) that simultaneously characterise and constrain the phenomenon and (3) the emerging pattern that arises from the complex web of determinants. The sports injuries literature has long sought for some of these features. However, the existing information about these features is still not organised according to a complex system framework that allows us to have some insights on injury prediction (despite its limitations).

To illustrate the complexity, let us consider the example of ACL injury, where there are some observed regularities that lead to its emergence. Figure 2A depicts the web of determinants for an ACL injury in basketball. For basketball athletes, the typical unanticipated environmental events (UEE) ${ }^{44}$ the presence of $\mathrm{DKV}^{44}$ and hip weakness (HW) are the main elements comprising the web of determinants. These elements take part in most of the existing interactions (unidirectional or bidirectional relationships) and, as such, influence and are influenced by other elements. In this case, the interacting configuration could be pictured as follows: the presence of DKV is influenced by fatigue,${ }^{44}$ hip muscle strength, neuromuscular control (ability to properly recruit the required muscles), foot complex anatomical alignment and training load. These variables are themselves modulated by other factors such as age and sex (eg, influence of age on hip muscle strength). ${ }^{45}$ In addition, training load influences the attention level and anxiety, which may increase fatigue and, by this connection, increase DKV. The manner in which these interactions play and how they together influence the occurrence of an ACL injury will create a risk profile (regularity) that is specific for the analysed context.

A single risk factor (ie, DKV) does not warrant the occurrence of the injury. On the other hand, the identification of the risk profile may inform about the probability of the injury occurrence. Since injury is a complex phenomenon characterised by uncertainties and inherent non-linearity, an ACL injury will emerge when a specific pattern of interaction happens in the presence of an inciting event of a given value. Thus, the best manner to predict an injury is by understanding the interactions among the web of determinants and not the determinants themselves.

As another example, figure 2B depicts the web of determinants of an ACL injury in ballet dancers. Owing to the type of training received and the need for movement quality, these athletes do not have, as the main elements, the presence of DKV and $\mathrm{HW}^{46}$ In addition, in ballet, UEE are rare when compared to collective sports. ${ }^{46}$ In this sense, fatigue, attention level, anxiety and, possibly, other unidentified variables may be the main interacting units of the web of determinants. Fatigue, in turn, is modulated by the amount of training sessions, which will also influence other psychological factors that may be differently influenced by sex. In the presence of a specific interacting pattern, the level of fatigue may alter the quality of movement and lead to the occurrence of DKV, which may produce an ACL injury. The web of determinants for ballet dancers has a different configuration of interactions among factors, when compared to basketball players. Similar factors have different contributions (weights) and interact differently with other factors, as the context of practice also differs. However, in each context, the observance of a given interacting pattern or risk profile will be related to a given probability of ACL occurrence.

Instead of looking for the units (first level risk factors), we should look for the existing pattern of interactions among the units (regularities). Only after we learn about those regularities, the identification of the relationships between contributing units (second level risk factors) will be necessary. This approach will allow the development of effective interventions to address the
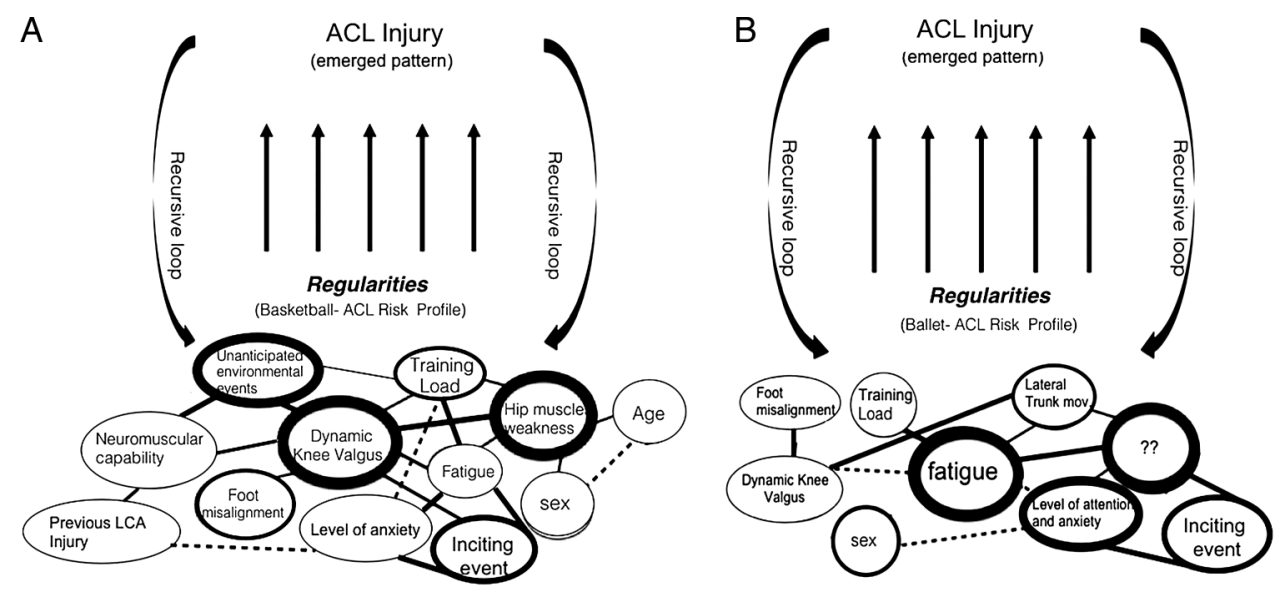

Figure 2 (A) Web of determinants for an $A C L$ injury in basketball athletes and (B) web of determinants for an $A C L$ injury in ballet dancer. 
identified risk profile. Therefore, injury prediction, as in a stock market and weather forecast, should rely on the probability that the presence of observable regularities (risk or protective profile) may be associated with the emergence of a given injury.

\section{CONCLUSION}

Recently, a complex systems approach has been used to predict complicated problems in medicine, biology, economics and social science. This conceptual paper serves as a challenge and as a support, as it indicates the weaknesses of the established model of thinking and proposes a model, which moves us from trying to find 'causes' to finding 'relations' that support the emergence of a sports injury. The limitation of our 'explanation power' of complex phenomena should not prevent us from trying to improve our 'predictive power' for injury occurrence. Adopting the complex systems approach may push us forward in terms of concepts and methods to improve sports injury prediction. In this sense, moving research from isolated risk factors to injury pattern recognition, by means of identification of the complex pattern of interactions among the web of determinants, is obligatory. Although difficult, it is feasible to identify and even understand the regularities of a web of determinants using real data and statistical modelling. This approach may be the only option if we accept the non-linearity and complexity of sports injury.

\section{What are the findings?}

- Sports injuries are complex emergent phenomena, produced by interactions among different units (web of determinants), which may produce regularities (risk profile) that prompt the emerging pattern (injury).

- Sports injury prevention relies on the identification of risk profiles, which means moving from risk factors to risk pattern recognition. This approach considers an interconnected and multidirectional interaction between all factors, which embrace the complex nature of the sports injury.

- Improvements in sports injuries prediction, as well as in prevention, depend on coherence among the phenomena of interest (sports injuries as an emergent event), philosophical paradigm (complexity) and methods of analysis. In this sense, non-linear and complex system approaches should be explored.

\section{How might it impact on clinical practice in the near} future?

- Clinicians should be aware of how risk factors may interact, rather than list several isolated risk factors, in order to plan effective preventive intervention.

- Risk profile may include non-linear interaction between risk factors from different scales, such as biomechanical, training characteristics, psychological and physiological. Additionally, risk profile should be continuously assessed throughout preseason and in season.

- The recognition of the web of determinants in clinical practice might include risk factors that strongly influence the outcome and interact in many different ways with several variables.
Acknowledgements The authors would like to acknowledge Dr Roberto Bittencourt and Dr Rosana Sampaio for introducing the role of complexity in health.

Contributors NFNB, LDM, JMO and STF contributed to the manuscript's conception and design. AN-A contributed to the analyses and interpretation of the statistical session, accurate and critical revision of the manuscript as well as approval of the final version. NFNB was the primary author and was responsible for the initial preparation of the manuscript. WM and STF contributed to the accurate and critical revision of the manuscript as well as approval of the final version. All authors were involved in the preparation and editing of the manuscript.

Funding The Minas Gerais state research-funding agency FAPEMIG and the Brazilian research-funding agencies CAPES and CNPq have supported this study.

Competing interests None declared.

Patient consent Obtained.

Provenance and peer review Not commissioned; externally peer reviewed.

\section{REFERENCES}

1 van Mechelen W, Hlobil H, Kemper HC. Incidence, severity, aetilogy and prevention of sports injuries. A review of concepts. Sports Med 1992;14:82-99.

2 Quatman CE, Quatman CC, Hewett TE. Prediction and prevention of musculoskeletal injury: a paradigm shift in methodology. Br J Sports Med 2009;43:1100-7.

3 Plsek P, Greenhalgh T. Complexity science: the challenge of complexity in health care. BMJ 2001;323:625-8.

4 Mendiguchia J, Alentorn-Geli E, Brughelli M. Hamstring strain injuries: are we heading in the right direction? Br J Sports Med 2012;46:81-5.

5 van der Worp $H$, van Ark M, Roerink $S$, et al. Risk factors for patellar tendinopathy: a systematic review of the literatute. Br J Sports Med 2011;45:446-52.

6 Coffey DS. Self-organization, complexity and chaos: the new biology for medicine. Nat Med 1998;4:882-85.

7 Rickles D, Hawe P, Shiell A. A simple guide to chaos and complexity. J Epidemiol Community Health 2007;61:933-7.

8 Philippe P, Mansi O. Nonlinearity in the epidemiology of complex health and disease processes. Theor Med Bioeth 1998;19:591-607.

9 Meeuwisse WH, Tyreman H, Hagel B, et al. A dynamic model of etiology in sport injury: the recursive nature of risk and causation. Clin J Sport Med 2007;17:215-19.

10 Holland JH. Hidden order: how adaptation builds complexity from chaos. Redwood City, USA: Addison-Wesley Longman Publishing Company, 1995. ISBN: 0-201-40793-0.

11 Hulme A, Finch CF. From monocausality to system thinking: a complementary and alternative conceptual approach for better understanding the development and prevention of sports injury. Inj Epidemiol 2015:2-31.

12 Galea S, Riddle M, Kaplan GA. Causal thinking and complex system approaches in epidemiology. Int J Epidemiol 2010;39:97-106.

13 Doll R, Hill AB. A study of the aetiology of carcinoma of the lung. $\mathrm{Br}$ Med J 1952;13:1271-86.

14 Hewett TE, Myer GD, Ford KR, et al. Biomechanical measures of neuromuscular control and valgus loading of the knee predict anterior cruciate ligament injury risk in female athletes: a prospective study. Am J Sports Med 2005;33:492-501.

15 Krosshaug T, Nakamae A, Boden BP, et al. Mechanism of anterior cruciate ligament injury in basketball: video analysis of 39 cases. Am J Sports Med 2006;35:359-67.

16 Malliaras P, Cook JL, Kent P. Reduced ankle dorsiflexion range May increase the risk of patellar tendon injury among volleyball players. J Sci Med Sports 2006:9:304-9.

17 Doll WE Jr, Trueit D. Complexity and the health care professions. J Eval Clin Pract 2010;16:841-8

18 Weaver W. Science and Complexity. Am Sci 1948;36:536-44

19 Higgins JP. Nonlinear systems in medicine. Yale J Biology Medicine 2002;45:247-60.

20 Farmer D, Gallegati M, Hommes C, et al. A complex systems approach to constructing better models for managing financial markets and the economy. Eur Phys J Special Topics 2012;21:295-324.

21 Rea TJ, Brown CM, Sing CF. Complex adaptive system models and the genetic analysis of plasma HDL-cholesterol concentration. Perspect Biol Med 2006:49:490-503.

22 Pfeiffer $M$, Hohmann A. Applications of neural networks in training science. Hum Mov Sci 2012;31:344-59.

23 Emery CA. Identifying risk factors for hamstring and groin injuries in sport: a daunting task. Clin J Sports Med 2012;22:75-7.

24 Ekstrand J, Hägglund $M$, Waldén $M$. Injury incidence and injury patterns in professional football: the UEFA injury study. Br J Sports Med 2011;45:553-8.

25 von Bertalanfly L. General system theory: foundations, development, applications. Penguin university books. New York, USA: George Braziller Inc., 1969.

26 Rosen R. Essays of life itself. New York, USA: Columbia University Press, 2000. ISBN: 0-231-10511-8 
27 von Bertalanffy L. The theory of open systems in physics and biology. Science 1950;111:23-9.

28 Haken H. Synergetics: are cooperative phenomena governed by universal principles? Naturwissenschaften 1980;67:121-8.

29 Bolgla LA, Malone TR, Umberger BR, et al. Hip strength and hip and knee kinematics during stair descent in females with and without patellofemoral pain syndrome. J Orthop Sports Phys Ther 2008;38:12-18.

30 Bittencourt NF, Ocarino JM, Mendonça LD, et al. Foot and hip contribution to high frontal plane knee projection angle in athletes: a classification and regression tree approach. J Orthop Sports Phys Ther 2012; 42:996-1004.

31 Myer GD, Ford KR, Barber Foss KD, et al. The relationship of hamstrings and quadriceps strength to anterior cruciate ligament injury in female athletes. Clin J Sport Med 2009;19:3-8.

32 Freckleton G, Pizzari T. Risk factors for hamstring muscle strain injury in sport: a systematic review and meta-analysis. Br J Sports Med 2013;47:351-8.

33 Bahr R. Why screening tests to predict injury do not work-and probably never will...: a critical review. Br J Sports Med 2016;50:776-80.

34 Mais V, Yen SS. Prolactin-releasing action of gonadotropin-releasing hormone in hypogonadal women. J Clin Endocrinol Metab 1986:62:1089-92.

35 Firth WJ. Chaos—predicting the unpredictable. BMJ 1991;303:1565-8.

36 Niska H, Hiltunen T, Karppinen A, et al. Evolving the neural network model for forecasting air pollution time series. Eng App Artif Intell 2004;17: 159-67.

37 Serrano-Cinca C. Self Organizing Neural Networks for Financial Diagnosis 1996. Decis Support Syst 1996;17:227-38.
38 Hastie T, Tibshirani R, Friedman J. The elements of statistical learning. New York, USA: Springer, 2001.

39 Butchart C, Ismailoglu F, Myint PK, et al. Identification of possible determinants of inpatient mortality using Classification and Regression Tree (CART) analysis in hospitalized oldest old patients. Arch Gerontol Geriatr 2013;56:188-91.

40 D'alisa S, Miscio G, Baudo S, et al. Depression is the main determinant of quality of life in multiple sclerosis: a classification-regression (CART) study. Disabil Rehabil 2006:28:307-14

41 Auchincloss AH, Diez Roux AV. A new tool for epidemiology: the usefulness of dynamic-agent models in understanding place effects on health. Am J Epidemiol 2008;168:1-8.

42 http:goo.gl/veJ74Q (accessed Feb 2016).

43 Chipman HA, George El, McCulloch RE. BART: Bayesian additive regression trees. Ann App/ Stat 2010;1:266-98.

44 Liederbach M, Kremenic IJ, Orishimo KF, et al. Comparison of landing biomechanics between male and female dancers and athletes, part 2 influence of fatigue and implications for anterior cruciate ligament injury. Am I Sports Med 2014;42:1089-95.

45 Brent J, Myer GD, Ford KR, et al. The effect of sex and age on isokinetic hip abduction torques. J Sports Rehabil 2013;22:41-6.

46 Orishimo KF, Liederbach M, Kremenic IJ, et al. Comparison of landing biomechanics between male and female dancers and athletes, part 1: Influence of sex on risk of anterior cruciate ligament injury. Am J Sports Med 2014;42:1082-8.

$47 \mathrm{http}: / / w w w . t r o j a n m i c e . c o m / a r t i c l e s / c o m p l e x a d a p t i v e s y s t e m s . h t m$ (accessed Feb 2016). 